\title{
Diferenciación, especialización y exclusividad
}

\section{Differentiation, expertise and exclusivity}

\author{
Gustavo E. Mata Fernández-Balbuena \\ Ingeniero Industrial Superior \\ CEPADE. Industriales Escuela de Negocios. Universidad Politécnica de Madrid \\ gmatafb@gmail.com
}

Fecha de recepción: 29-10-2009
Fecha de aceptación: 18-06-2010

Resumen: ¿Se puede intentar ser diferente para todo un sector, como sostiene M.E. Porter, o la condición de diferenciación lleva implícito que el diferente sólo pueda serlo para una parte, es decir para un segmento? Para nosotros la respuesta es obvia, sólo se puede ser diferente para una parte. Si se fuera diferente para todos ¿Para quién se sería diferente? Puede que se fuera único, pero no diferente. En el artículo se trata de la condición de segmentabilidad de los sectores, la diferenciación en los mismos y la exclusividad; y se introduce un nuevo modelo espacial, complementario de la teoría de Edward Hastings Chamberlin de la competencia monopolística, que ilustra las ideas que se sostienen.

Palabras clave: sector de actividad, segmento, diferenciación, exclusividad, competencia monopolística, estrategias genéricas, identificación, diferenciación formal.

\begin{abstract}
Is it possible to try to be different for a whole sector, as M.E. Porter holds, or does the condition of differentiation entail that the difference can only be for a part, that is to say, for a segment? For us the answer is obvious: it is only possible to be different for a part. If we are different from all, from whom would we be different? One might be unique, but not different. The article deals with the condition of «segmentability» of the sectors, their differentiation and their exclusivity. We introduce a new space model, complementary of Edward Hastings Chamberlin's theory of the monopolistic competition, which illustrates the ideas that are hold in the matter.
\end{abstract}

Key words: industry, segment, differentiation, exclusivity, monopolistic competition, generic strategies, identification, formal differentiation.

\section{Sectores en el que los productos son diferenciados}

En un sector en el que hubiera diferenciación, cada segmento del mercado percibiría de forma diferenciada los distintos productos, de forma que cada modalidad específica de producto sería la preferida para cada segmento diferenciado que consideraría a los demás productos sustitutivos próximos pero no perfectos del preferido. Un sector en el que los productos fueran diferenciados implicaría, para nosotros, que habría segmentos de mercado identificables en él. Sin segmentos no hay diferenciación posible.

En cada sector el criterio o los criterios relevantes para identificar los segmentos serán unos u otros, pero, en cualquier caso, la característica común de un mercado así sería que cada segmento de mercado sería susceptible de ser atendido, de forma ventajosa, con una propuesta específica de producto adaptado a su necesidad específica diferenciable.

\section{Productos sustitutivos. Los límites del sector con productos diferenciados. El concepto de grupo estratégico}

Para poder analizar el papel de la diferenciación del producto en el sector, deberíamos considerar, en primer lugar, cómo se puede definir un sector con productos diferenciados. Consideraremos, como hace Porter, que los sustitutivos más próximos y homo- 
géneos son un grupo estratégico -que será para nosotros el sector de actividad en sentido estricto- y los grupos de sustitutivos más alejados serán considerados los productos sustitutivos -también en sentido estricto y diferenciando, a su vez, el papel de cada grupo estratégico de sustitutivos-. Esto permitiría analizar el problema por fases; primero, la diferenciación dentro de cada grupo relativamente homogéneo -el grupo estratégico- $y$, segundo, la interrelación entre los distintos grupos o diferenciación entre los grupos.

\section{Diferenciación real y diferenciación formal: la identificación de productos similares a través de las marcas comerciales}

Al considerar las causas de la diferenciación, se presenta un asunto de trascendental importancia -como tendremos ocasión de analizar también más adelante- que trastocaría las conclusiones de cuanto vamos a analizar; hay una diferenciación, que podemos llamar, real y una diferenciación sólo formal: la creada a través de la publicidad, que, además de dar a conocer los nuevos productos, sirve a la identificación formal de los mismos a través de las marcas: productos iguales, o perfectamente equivalentes, se identifican a través de las marcas, en cuya creación se invierten cantidades masivas de dinero; estas inversiones no van destinadas a satisfacer mejor la necesidad, es decir, a «producir» el producto, sino a modificar la demanda del mismo, condicionando tanto la demanda de la clase de producto anunciada -la amparada por la marca-, como también la demanda de todos los demás de la categoría, modificando hacia arriba la demanda de la marca y del conjunto, y hacia arriba o hacia abajo la demanda de cada uno de los demás integrantes del sector, así como incrementando el poder de monopolio relativo ${ }^{2}$ de la marca anunciada frente a su segmento de mercado.

Cuando hablemos de sectores en los que haya diferenciación, nos estaremos refiriendo a sectores que estarían configurados por un grupo de productos que serían sustitutivos próximos entre sí, y que lo serían «per se» y no por efecto de la identificación de la marca. No estamos incluyendo en el análisis los costes y los efectos de la publicidad.

\section{La teoría de la competencia monopolística}

La aproximación teórica más rigurosa a lo que supone la diferenciación de los productos es la teoría de la competencia monopolística de Edward Hastings Chamberlin ${ }^{3}$. Aunque en cualquier manual de microeconomía avanzada se alude a esta teoría, no se suele incluir una explicación suficiente de la misma a efectos micro-económicos, resaltando sólo la consecuencia macro-económica de la pérdida de eficiencia económica global que supone la diferenciación respecto de la competencia perfecta: más competidores y precios más altos, aunque no necesariamente beneficios para los productores. Por ello, nos permitimos desarrollar brevemente esta teoría, pues, en nuestro enfoque, va a resultar crítica para comprender a fondo el papel de la diferenciación como barrera y, porque, apoyándonos en ella, desarrollaremos algunos sencillos modelos espaciales originales que nos van a llevar al nuevo concepto clave para entender lo que puede provocar la diferenciación: lo que hemos dado en llamar segmentos estancos.

En un sector en competencia perfecta, con producto indiferenciado y un número suficientemente grande de compradores y de vendedores, ningún competidor podría vender, ni siquiera una unidad, a un precio superior al de mercado; también si bajase el precio sólo él, podría quedarse, si tuviera capacidad para abastecerla, con toda la demanda. En definitiva, cada concurrente se enfrentaría a una curva individual de demanda plana, perfectamente elástica, que pasaría por el precio de mercado. No es fácil encontrar mercados así en la práctica, salvo en algunos sectores agrícolas o ganaderos que producen «commodities», que se aproximan grandemente.

En un sector en competencia monopolística los productos serían diferenciados; para cada segmento de mercado habría una modalidad de producto que sería preferible a los demás y cada productor tendría un cierto poder de monopolio sobre su mercado próximo; es decir, si un productor subiera algo el precio, manteniéndolo los demás, éste no perdería todas sus ventas, sino sólo una parte, y si lo bajase, tampoco se quedaría con toda la demanda aunque aumentarían algo sus ventas. Hay muchos sectores reales que se aproximan a este modelo teórico.

\footnotetext{
2 El índice de Lerner: mide el poder de monopolio. Abba P. Lerner. 1903, 1982, «The Concept of Monopoly and the Measurement of Monopoly Power», The Review of Economic Studies, Vol. I, No. 3(Jun., 1934), pp. I57- I75. Abba P. Lerner. 1903, 1982.

3 Edward Hastings Chamberlin "Theory of Monopolistic Competition, Harvard University Press, Cambridge, MA, USA I946"
} 
Esa suerte de poder relativo de monopolio que cada competidor tendría sobre su segmento de mercado más cercano, significaría, en cierto modo, que un competidor monopolístico se enfrentaría a dos diferentes curvas de demanda: la que se derivaría de que todos los competidores -incluido él- modificaran al unísono sus precios, y la que se derivaría de que sólo los modificara él y los mantuvieran los demás. Analicemos lo que ocurriría entonces.

Consideremos que DD' fuera la recta de demanda correspondiente a cada competidor en el caso de que todos movieran conjuntamente sus precios, es decir, ésta sería una fracción de la demanda global; consideremos también que para el resto de los concurrentes estuviera fijado el precio p y que, en esas condiciones, la recta de demanda para un competidor dado, si todos los demás, salvo él, insistimos, mantuvieran sus precios, fuera dd'; y consideremos, por fin, que la curva de costes medios de cada competidor fuera la de la Figura 1.

Figura I

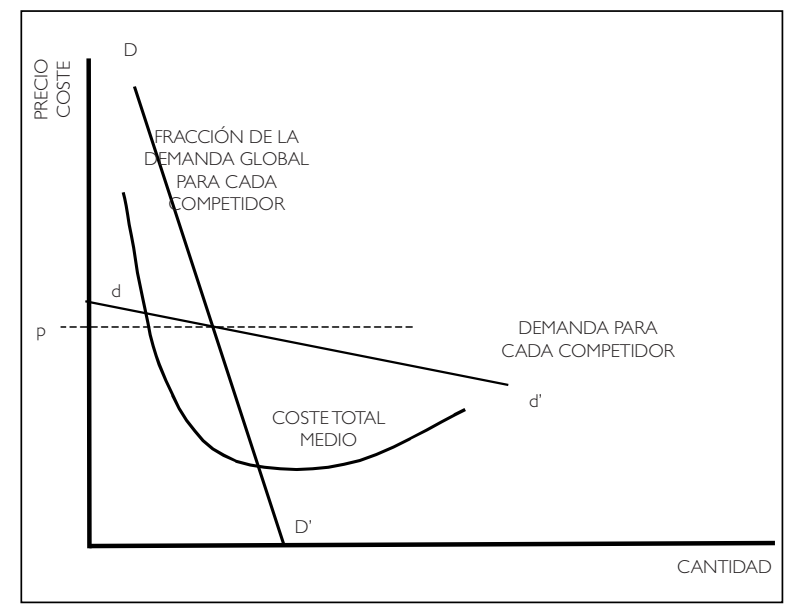

Cuando todos los concurrentes modificaran a la vez sus precios, la demanda para cada uno sería una fracción de la demanda global -la fracción enésima, si fueran n los concurrentes-; la recta de demanda para cada uno, DD', se iría desplazando hacia la izquierda a medida que fuera incrementándose el número de concurrentes o hacia la derecha si estos disminuyeran. Fijado un precio p para todos los demás, cuando uno solo modificara sus precios, la recta de demanda para él, dd', tendría una elasticidad, necesariamente mayor que la de la demanda anterior, tanto más parecida a ésta cuanto más diferenciada esté su oferta de la de los demás, para su segmento. Para cada precio, habría una recta de demanda dd'; todas ellas serían paralelas entre sí, y cada una de ellas cortaría a la recta de demanda de cada uno -si todos tuvieran el mismo precio- DD, en el punto del precio fijado para todos.

En estas condiciones, a cualquier competidor individual le merecería la pena bajar sus precios hasta un nivel p' (Figura 2) -en la intersección de su recta de ingreso marginal con su curva de coste marginal-, siempre que los demás mantuvieran los suyos, para maximizar sus beneficios. Si la porción de mercado que arrebatase a los demás se repartiera por igual entre todos, y éstos fueran muchos, podría esperase que mantuvieran su precio, pues apenas se habrían enterado de la disminución de sus ventas; pero, aún así, lo mismo que a uno le merecería la pena bajar el precio para aumentar sus ganancias también le merecería la pena hacerlo a los demás y, siendo así, sin duda lo harían, con lo que, cuando todos lo hicieran, la cantidad vendida por cada uno al precio $p^{\prime}$, se reduciría hasta q"'.

Figura 2

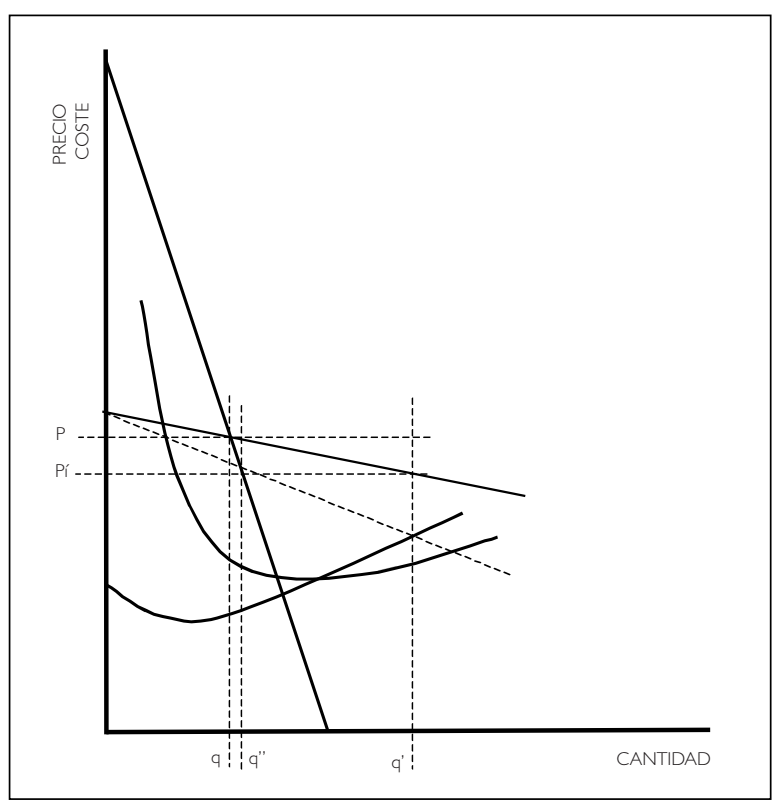

Ahora (Figura 3), la recta dd' para cada competidor se habrá desplazado hacia abajo, puesto que el nuevo precio fijado sería p' y la recta dd ' habría de pasar por el precio fijado en el sector. De nuevo, para estas nuevas condiciones, cada competidor podría aumentar sus beneficios si redujese los precios, lo que le llevaría a hacerlo, hasta maximizarlos para un precio p" y una cantidad q"'. Pero, también ahora, esa condición sería la misma para todos, así que todos reducirían sus precios, con lo que la cantidad vendida por cada uno al precio $\mathrm{p}^{\prime \prime}$, se reduciría hasta $\mathrm{q}^{\prime \prime \prime}$.' 
Figura 3

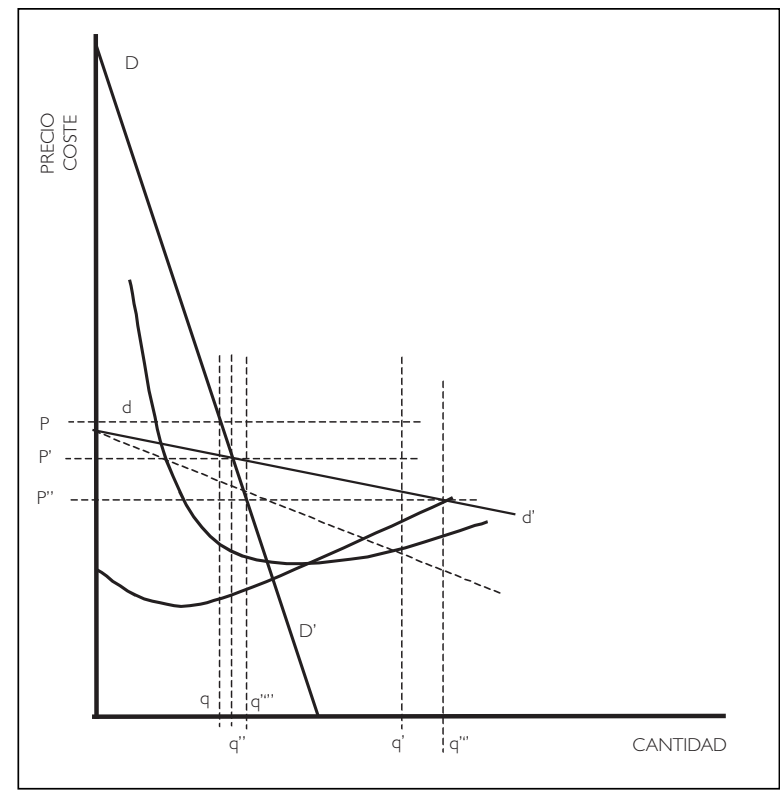

Reiterado el proceso, la recta de demanda dd' se estaría desplazando sobre la recta de demanda DD', hasta que se alcanzara un precio por debajo del cual ya no sería posible tener beneficios; el correspondiente a la intersección de la recta DD' con la curva de costes medios (Figura 4).

Figura 4

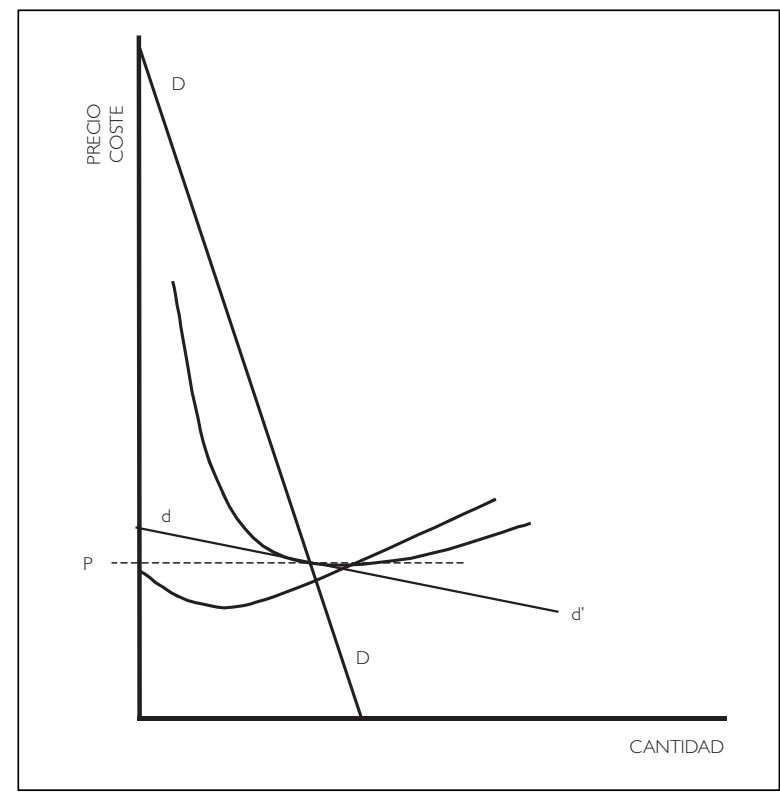

Debemos precisar que el hecho de que, en la Figura 4, la recta DD' corte a la de costes medios en el punto de tangencia entre dd' y esa misma curva de costes medios, significa que, en este caso, la condición de equilibrio es compatible con el número de competidores instalados. No tiene por qué ser necesariamente así. Analizaremos el papel del número de competidores a continuación y comprenderemos mejor lo que estamos diciendo.

Pensemos que mientras el proceso de ajuste hacia el equilibrio no se haya completado, el sector -en beneficios- atraerá a nuevos competidores, que se instalarían. ¿Hasta qué limite?: hasta que la recta DD', que como sabemos se desplaza hacia la izquierda con cada entrada de un nuevo competidor, sea tangente a la curva de costes medios. (Figura 5)

Figura 5

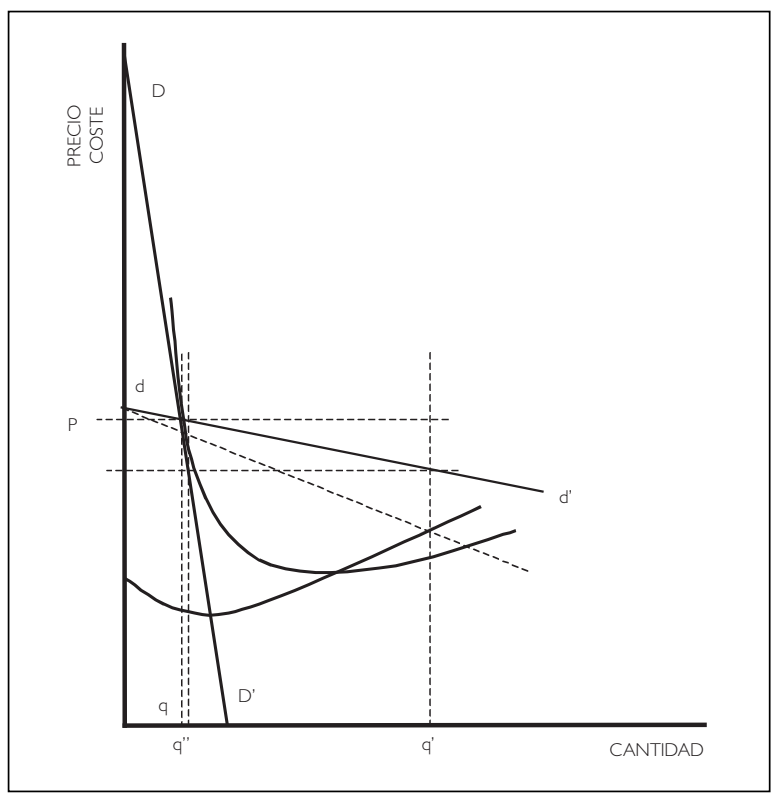

Si así fuera, ninguno de los competidores podría tener beneficios; el precio común, al que ninguno tendría pérdidas, sería p, el correspondiente al punto de tangencia, ofertando cada uno de ellos una cantidad q. Pero en éste punto no habría verdadero equilibrio: puesto que cada concurrente tendría la oportunidad de tener beneficios, siempre que los demás mantuvieran ese precio, bajando el suyo hasta el nivel p', lo haría, ofertando la cantidad q'; claro que, como todos tienen esa oportunidad, todos lo harían con lo que la cantidad vendida por cada bajaría hasta q"', lo que les llevaría a todos a pérdidas. Con todo cada uno de ellos seguiría pensando que puede reducir éstas pérdidas, e incluso tener beneficios, bajando de nuevo los precios, por lo que el proceso continuaría, hasta que las pérdidas produjesen salidas de alguno de los concurrentes desplazándose entonces la recta DD' hacia la derecha. 
Vemos pues que el efecto conjunto de ambas tendencias analizadas -entrada y salida de competidores sin restricciones, según haya beneficios o pérdidas y posibilidad de incrementar beneficios bajando individualmente el precio- conduce a un equilibrio en torno al punto de tangencia entre dd' y la curva de costes medios; y a que la recta DD corte a la de curva de costes medios, sensiblemente, en ese punto -tanto más exactamente cuanto mayor número de concurrentes haya en la situación de equilibrio-. (Figura 6.)

Figura 6

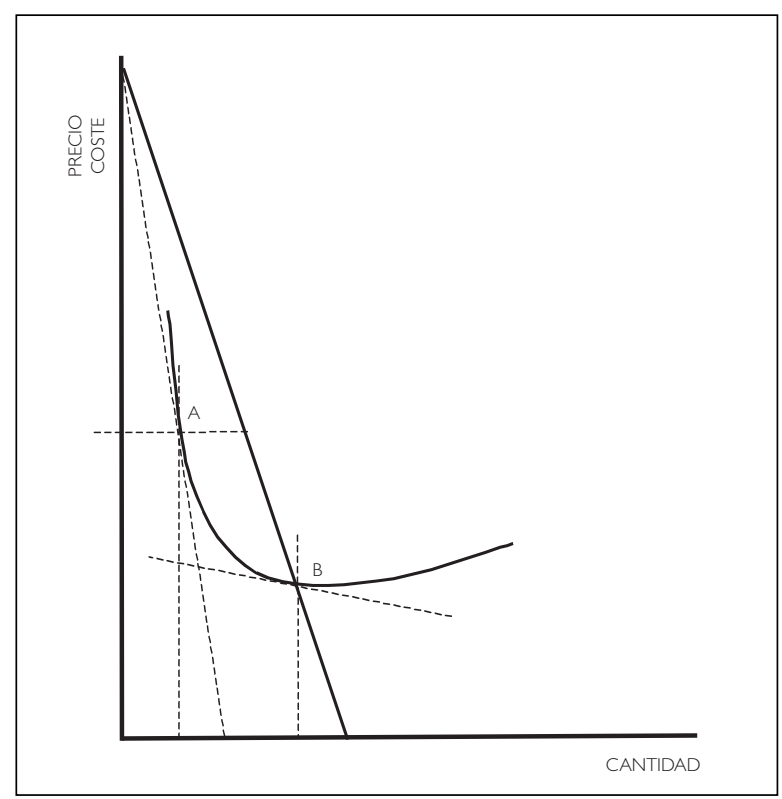

\section{El reconocimiento de la mutua interdependencia: el pequeño grupo en competencia monopolística}

Pero, ¿qué ocurriría si los concurrentes en lugar de pensar en que no son interdependientes, supieran que lo son? Esto no es probable que ocurra a menos que el número de competidores del grupo sea pequeño; entonces será más fácil que todas las empresas reconozcan su mutua interdependencia. Si así fuera, y cada uno accediera a una demanda parcial como DD', mantendrían sus precios en el nivel p, restringiendo su oferta hasta el nivel q, con lo que todos maximizarían sus beneficios. (Figura 7)

Claro que cuando hubiera beneficios, éstos atraerían a nuevos concurrentes, desplazando entonces la recta de demanda DD de cada uno hacia la izquierda
(Figura 8), hasta que el ingreso de uno más llevara a todos a la situación de pérdidas. Eso podría dar lugar a que haya beneficios para los instalados y esté dificultada la entrada de competidores potenciales; es decir a barreras de acceso en el sentido de Bain ${ }^{4}$.

Figura 7

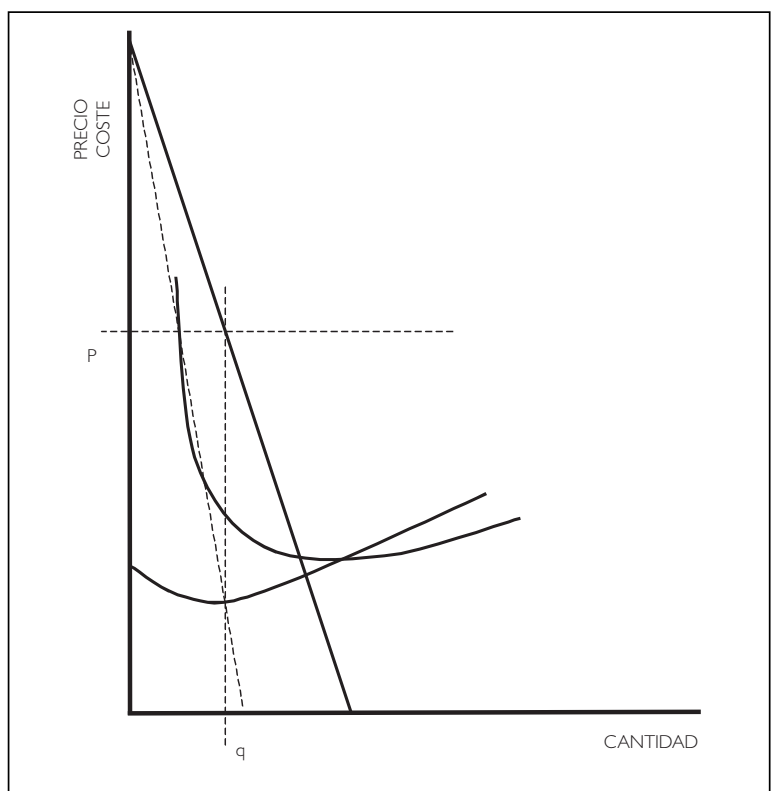

Figura 8

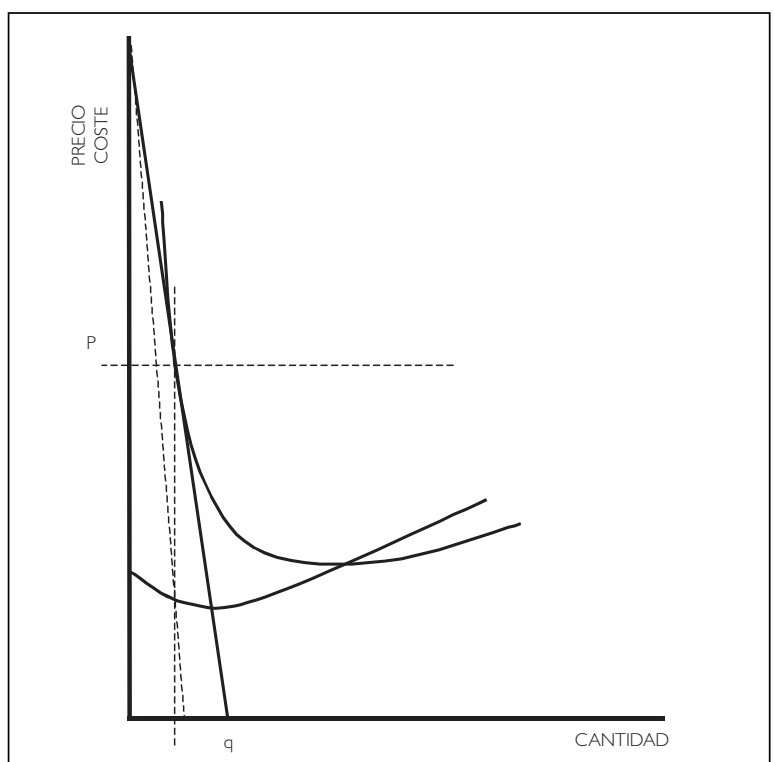

Cuando haya reconocimiento de la mutua interdependencia entre los competidores del grupo -es de-

\footnotetext{
${ }^{4}$ J. S. Bain "Barriers to New Competition: their character and consequences in manufacturing industries", 1956. Harvard University Press, Boston, MA, USA
} 
cir un grupo pequeño-, el precio de equilibrio ya no tendría que ser necesariamente $B$; este equilibrio podría establecerse en cualquier punto entre ambos límites $A$ y $B$, que fuera compatible con un número determinado de competidores -naturalmente, no podría tratarse más que de un número entero de ellos. (Figura 9)

Figura 9

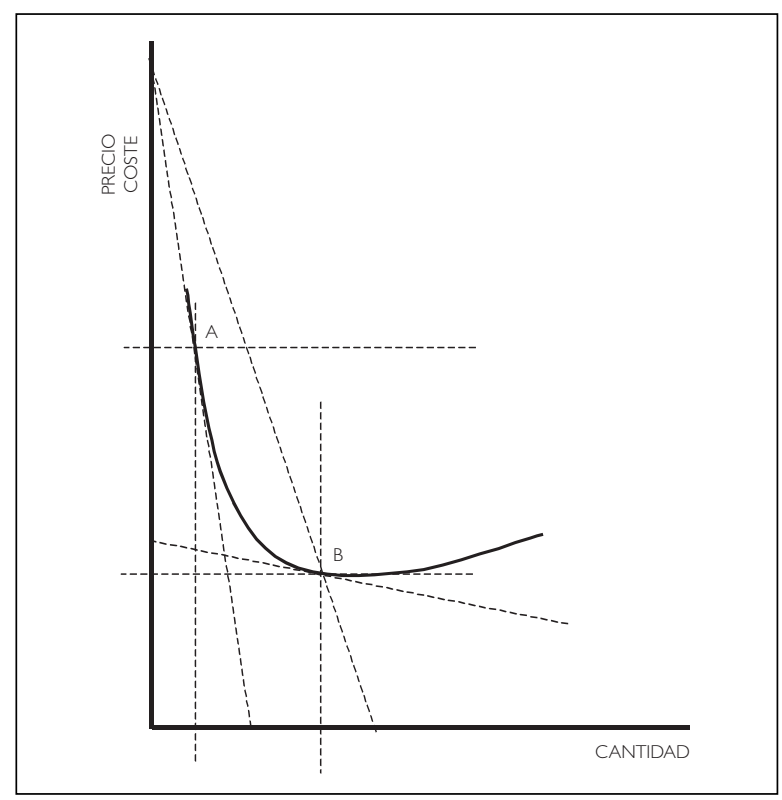

En la práctica, en muchos sectores de actividad, aun no siendo los competidores un grupo pequeño, se fijan los precios en un nivel que entienden razonable, más que en un nivel competitivo, que correspondería al que fijarían si estuvieran especulando sobre cuanto más venderían si los bajasen. Incluso, en determinadas actividades, se considera una práctica poco ética -denominada competencia desleal-bajar los precios por debajo de unas determinadas tarifas.

En cualquier caso, pese a que hubiera precios superiores a los competitivos, no habría finalmente beneficios, pues, el hecho de que a esos precios hubiera beneficios atraería a competidores potenciales a la actividad hasta que éstos desaparecieran, a menos que hubiera barreras adicionales de otro tipo para el ingreso.

La diferenciación por sí misma -salvo en el caso del pequeño grupo que reconozca su interdependencia $y$ en donde haya beneficios para $n$ instalados y no quepan $n+1$ - no puede dar lugar a la creación de barreras, aunque los precios de equilibrio, en sectores con productos diferenciados, resulten siempre su- periores a los que corresponderían si el sector estuviera en competencia perfecta, es decir si los productos fueran indiferenciados: la diferenciación provoca que todos los concurrentes operen por encima de sus costes medios mínimos e infrautilicen su capacidad, lo que daría lugar a precios más altos y mayor número de competidores que los que habría en el caso de competencia perfecta, pero no a que haya beneficios.

\section{Un modelo original de competencia monopolística de origen espacial}

Vamos a estudiar, a continuación, algunos elementos relacionados con la formación de barreras para el acceso en condiciones de competencia monopolística, no analizados por E. H. Chamberlin en su teoría -los cita simplemente-: los modelos de competencia monopolística de origen espacial y asimilables y las relaciones entre los diferentes grupos próximos integrados por competidores monopolísticos -evidentemente más próximos entre sí, que lo que lo están los diferentes grupos-.

Hasta aquí, en todo el análisis que hemos hecho, estamos suponiendo que, en un sector en competencia monopolística, cuando un competidor baja el precio, mientras los demás lo mantienen, su incremento de ventas se detrae por partes iguales de cada uno de los concurrentes. Esto, en la realidad, en los sectores asimilables al modelo de competencia monopolística, no suele ser así: normalmente los incrementos de ventas para el que baja el precio se detraen de los más cercanos, aquellos más próximos, con atributos específicos parecidos a la variedad de producto considerada.

Supongamos que los competidores, iguales en todo lo demás, hubieran de situarse en cualquier punto de una circunferencia -como si de puestos de vendedores de perritos calientes alrededor de un lago se tratase- a lo largo de la cual estuvieran los diferentes clientes, repartidos de forma homogénea -como si fueran bañistas en la orilla de un lago-y que todos ellos tuvieran un comportamiento perfectamente equivalente como consumidores. Supongamos también, como decíamos, que los competidores tuvieran el mismo producto, los mismos costes y que su única fuente de diferenciación posible fuera la ubicación.

El primer productor que se instalara lo haría en cualquier punto de la circunferencia, para él la ubicación sería indiferente (Figura I0-A). Pero, ¿dónde lo haría el segundo?: instalándose al lado del primero acce- 
dería a la mitad del mercado y en el punto diametralmente opuesto (Figura I0-B) también accedería a la mitad del mercado; parecería indiferente una u otra localización, aunque, es seguro que preferirá la segunda; ipor qué?: está claro que si lo hiciera así se especializaría en un segmento y tendría a su mitad de mercado algo defendida, lo que no ocurriría en el caso de emplazarse al lado del primero. La razón de esa mejor defensa se debe a la diferenciación; ahora, el producto del otro no es un sustitutivo perfecto del suyo; para su segmento de mercado más próximo el suyo es preferible pues está más cerca y es menos gravoso desplazarse para los clientes; si el otro competidor bajara el precio ya no se quedaría con todo el mercado, como en competencia perfecta, habría una elasticidad cruzada entre ambos productos; la competencia se establecería sobre bases de competencia monopolística.

Figura 10

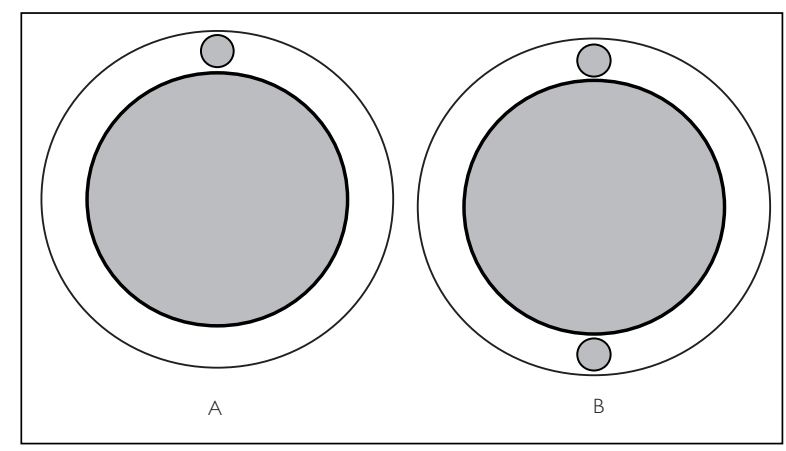

¿Dónde se instalaría un tercero? Razonemos un poco más allá: tanto si lo hiciera al lado de cualquiera de los instalados, como si lo hiciera en medio de ambos, sólo podría acceder a una cuarta parte del mercado y no a un tercio. (Figura I I). Pese a ser sólo tres concurrentes, para él sólo un cuarto del mercado estaría a su alcance preferente.

Figura I I

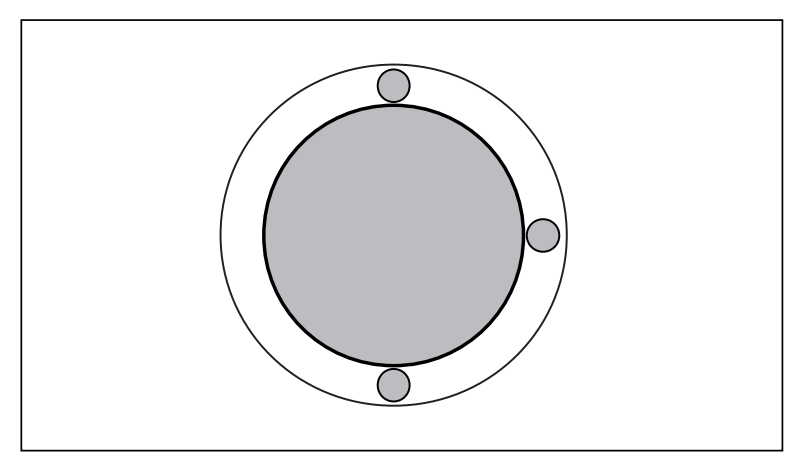

Imaginemos que la demanda disponible, tomando en cuenta las curvas de costes medios de cada uno de los competidores, diera para que se pudieran instalar tres concurrentes pero no cuatro (Figura 12).Tres, si se hubieran instalado todos en el mismo punto de forma indiferenciada, por ejemplo si hubiera una única ubicación autorizada, serían viables; pero ahora, diferenciados todos, el tercero ya no lo sería.

Figura 12

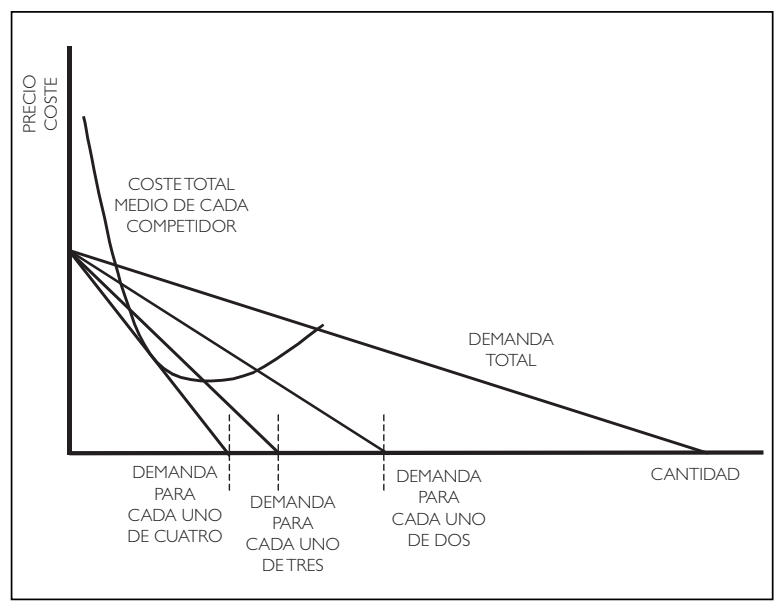

La barrera que antes, sin diferenciación, no existía para la entrada del tercero, sin embargo ahora, con los productos diferenciados, sí existiría; ahora no habría espacio suficiente entre los instalados para que cupiera el tercer competidor potencial. Las barreras por economías de escala se habrían potenciado como consecuencia directa de la diferenciación; los instalados tendrían mayores beneficios -serían solo dos y no tres a repartir los beneficios del cártel- y no por eso pondrían el acceso al mercado al alcance de los potenciales entrantes.

Ahora bien, para que la diferenciación tuviera algún efecto sobre las barreras, como acabamos de ver, tendría que haber costes fijos, si no hubiera costes fijos y el coste marginal fuera constante, no habría un tamaño eficiente. Pero si hubiera un tamaño eficiente -economías de escala hasta alcanzar ese tamañoy este fuera suficientemente grade para dar lugar a un grupo pequeño, el efecto de la diferenciación sería tal que, al diferenciar los segmentos de mercado, haría más eficaces en cada uno de los segmentos - de lo que serían en el mercado global- las barreras derivadas de las economías de escala, como acabamos de ver. Claro que para que fueran barreras eficaces haría falta, además, no sólo que el grupo que cupiera en cada segmento fuera pequeño sino que, reconociendo su mutua dependencia, actuara como un 
cártel. Sólo si hubiera economías de escala que pudieran actuar como barrera la diferenciación las potenciaría, sino no.

Las barreras que supondría la diferenciación de este tipo, tampoco serían necesariamente tan eficaces si el mercado estuviera creciendo, pues el constante desplazamiento hacia la derecha de las curvas de demanda disponibles para cada concurrente abriría finalmente el acceso a competidores potenciales que se convertirían en nuevos entrantes.

\section{La estanqueidad del segmento como condición límite de diferenciación}

Supongamos, siguiendo con el ejemplo anterior, que la demanda fuera tal que hubiera ocho competidores viables instalados a intervalos regulares alrededor de la circunferencia y que el precio común fuera p. (Figura |3)

Figura 13

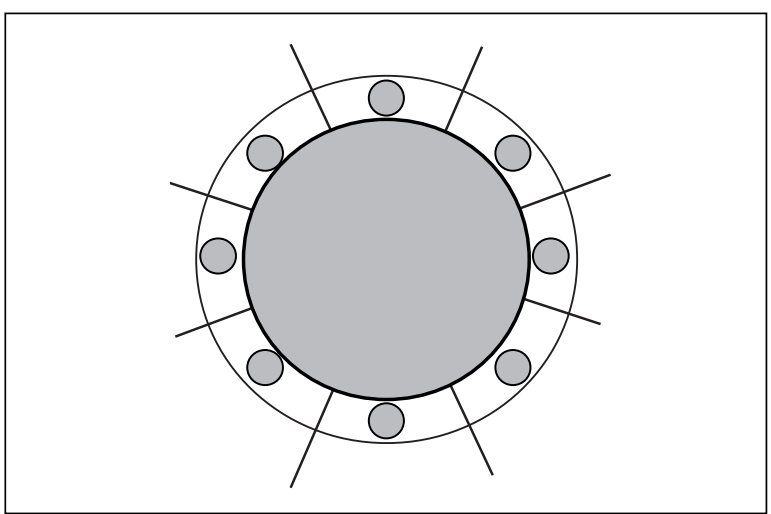

En este modelo, si uno solo de los competidores decidiera bajar su precio, y los demás lo mantuvieran, la bajada no afectaría a todos a la vez como en el modelo de la competencia monopolística de Chamberlin; afectaría en primer lugar a la demanda de los próximos, sin afectar nada a los más alejados, para, progresivamente, ir afectando a los demás a medida que se siguiera bajando. También si se subiera el precio, se verían afectados en primer lugar los más próximos, que serían quienes se repartirían el decremento de ventas del que hubiera subido el precio. Recordemos que la razón por la que, para cada grupo de clientes, un producto es preferible es la necesidad de desplazarse más lejos para conseguir cualquier otro; consideremos que el coste necesario para desplazarse la distancia que hay entre cada dos de los ocho concurrentes fuera t. En este supuesto, cuando uno solo de ellos hubiera bajado el precio desde $p$ hasta $p-t / 2$, manteniendo el precio los demás, los dos más próximos habrían perdido la mitad de sus ventas y él tendría la cuarta parte del mercado; si siguiera bajando, cuando hubiera bajado hasta $\mathrm{p}$-t, los dos más próximos habrán perdido todas sus ventas y, a partir de aquí, empezarían a perderlas los dos siguientes, de modo que cuando el precio llegase a $p$ $3 t / 2$ habrían perdido la mitad de las mismas, y el competidor que estuviese bajando los precios tendría ya la mitad del mercado; a partir de ahí las bajadas empezarían a afectar a los dos siguientes hasta que a un precio $\mathrm{p}-5 \mathrm{t} / 2$ el que estuviera bajando tendría tres cuartas partes del mercado y a un precio $p-3 t$ ya no venderían nada; finalmente, si continuara la bajada, el instalado en el extremo opuesto, que no se habría visto afectado hasta ahora, empezaría a estarlo, hasta que para un precio $p$ - 7/2t, tampoco éste podría vender nada al precio $\mathrm{p}$.

La recta $\mathrm{dd}^{\prime}$, correspondiente a la demanda de uno cuando sólo él modifica sus precios, sería, por tanto, la de la Figura 14.

Figura 14

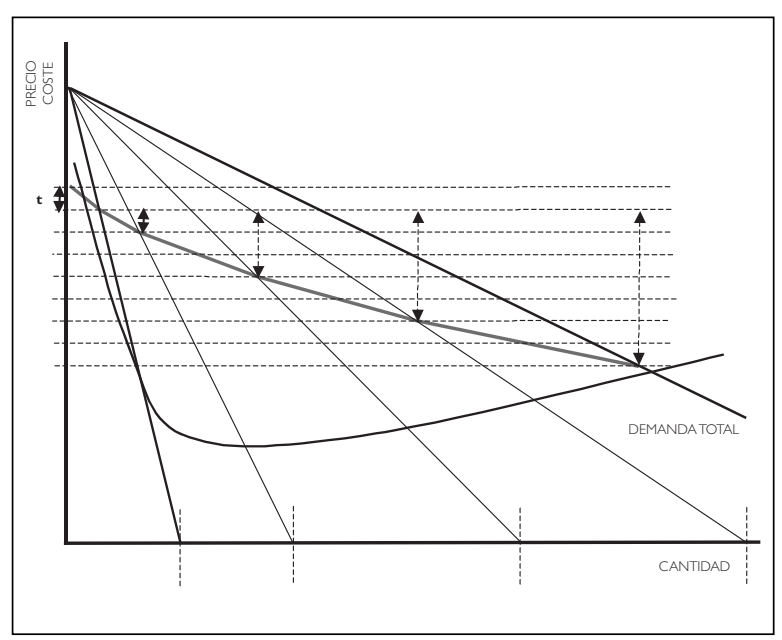

No pretendemos analizar ahora si el supuesto comportamiento de los demás -no reaccionar-sería razonable; ni tampoco si las bajadas le convienen al que baja para optimizar sus beneficios; ni hasta dónde le conviene bajar el precio; sólo pretendemos estudiar el efecto que tendría sobre sus ventas si lo hiciera y los demás no reaccionaran. En la realidad, en cuanto los más próximos se vieran afectados por la bajada, parece lógico que bajasen también los precios al mismo nivel que los hubiera bajado él; entonces, los siguientes también se verían afectados y harían lo propio, con lo que el efecto sería que el nuevo precio se 
instalaría rápidamente entre todos ellos, por lo que los precios y las cantidades vendidas por cada uno de ellos bajarían por fracción octava de la recta de demanda DD'.

Sigamos con el ejemplo. Para costes de transporte más altos, cada concurrente tendría mayor poder de monopolio sobre su segmento: los sustitutivos serían menos perfectos, la recta dd' sería más inelástica. (Figura 15).

Figura 15

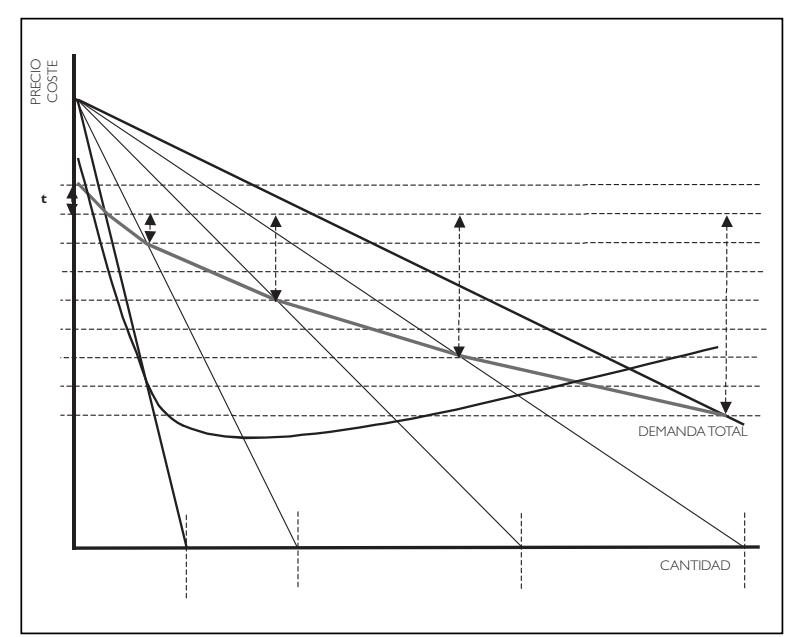

Cuanto mayores fueran los valores del coste del transporte t habría más diferenciación entre los diferentes concurrentes; incluso, para un cierto nivel de coste de transporte el segmento de mercado más alejado ya no estaría al alcance de las bajadas de sólo uno de los concurrentes; aunque por el efecto contagio la bajada podría acabar afectándoles a todos. (Figura 16)

Figura 16

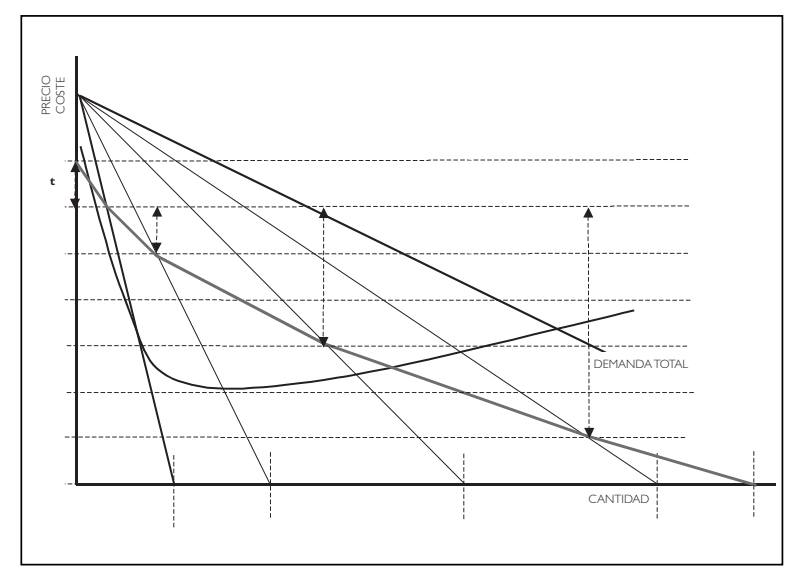

Llegará un momento, para un determinado valor de t, en que cada uno de los segmentos de mercado estaría cautivo de su suministrador más próximo; entonces dd' coincidiría con DD' y las bajadas de precio en el segmento no afectarían a los otros segmentos. (Figura 17).

Figura 17

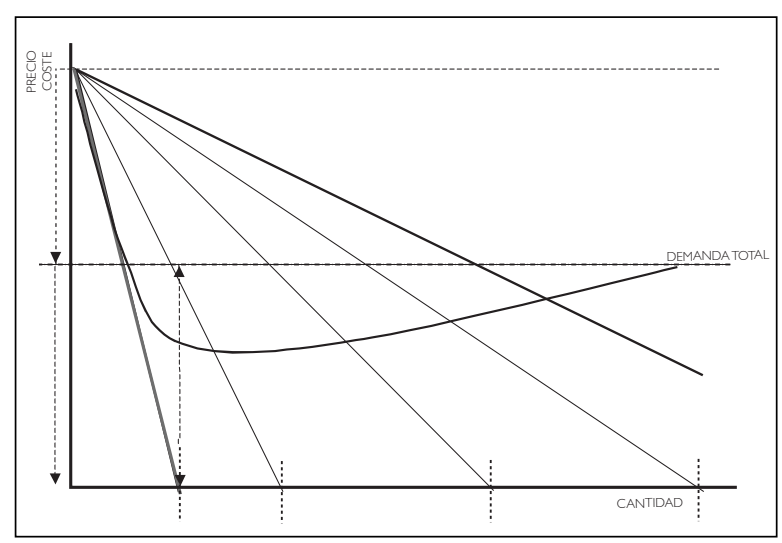

Esta es la condición de estanqueidad, una diferenciación tal que alcanza a eliminar la interrelación del segmento con los demás; ahora los productos ya no serían sustitutivos y cada segmento sería estanco. La demanda global del sector, como tal, sería una entelequia, estaría repartida entre segmentos estancos. Cada segmento sería, más bien, un sector en sí. La estanqueidad de los segmentos de un mercado sería, para nosotros, la condición extrema de la diferenciación. Cuando la diferenciación, que hace que, en cada segmento del mercado, cada producto sea preferido a los sustitutivos próximos, sea tal que el preferido no tuviera ya sustitutivos eficaces se habría dado la condición de estanqueidad en el segmento. En sectores segmentados en segmentos estancos no tiene sentido hablar del sector como un conjunto sino hablar de cada segmento; no habría, en sentido estricto, cuota de mercado global, sino cuota en cada segmento.

Frente a una situación así, el productor, si estuviera solo en su segmento, podría actuar como un monopolista y fijaría un precio que hiciera máximos sus beneficios, lo que podría atraer a otros con los que, de producirse la entrada, debería compartirlos. Claro que si las economías de escala no permitieran la entrada, como ya vimos -es decir, cuando el desplazamiento de DD hacia la izquierda deje a la recta de demanda sin cortar a la curva de costes medios-, se produciría una barrera. 


\section{La exclusividad percibida}

Porter considera la exclusividad percibida como la condición extrema de la diferenciación ${ }^{5}$. Esto resulta, cuando menos, confuso.

¿Qué es la exclusividad? Una cosa es el atributo del mercado: la segmentabilidad, que exige diferenciación de los productos que deben adaptarse a cada segmento; otra cosa es que esa segmentabilidad pueda generar segmentos estancos y que los productos de cada segmento no sean sustitutivos unos de otros; y otra cosa es la exclusividad. Puede que en cada segmento, por economías de escala, estanco en parte o en todo, quepa uno o unos pocos competidores. ¿Es eso exclusividad? Exclusivo es el que excluye; pero ¿en dónde?, ¿en todo el mercado porque nadie puede producir el producto porque éste es único, inimitable?; ¿o en un segmento del mercado, porque la propuesta -el producto- está en un segmento estanco y otro no cabría, no porque el producto sea inimitable sino sencillamente porque dos no caben?

Para nosotros, como hemos visto, la condición de diferenciación Ilevada al extremo sería la estanqueidad en el segmento. La exclusividad no estaría ligada a la diferenciación de los productos en el sector - que, como sabemos, daría lugar a que cada producto, para cada segmento, fuese el preferido y los demás fuesen sustitutivos no perfectos, lo que, en el caso extremo, produciría la eliminación de los sustitutivos cercanos, aunque no la existencia de competidores en el segmento- sino a la imposibilidad de que otros produjeran el producto lo que implicaría la imposibilidad de la existencia de competidores. Una cosa es que cada segmento sea en realidad un mercado estanco, aislado, y no haya productos sustitutivos y otra que un producto no pueda tener competidores por ser único y, en consecuencia también exclusivo, condición que se daría para el mercado global y no para un segmento. La exclusividad, entendida como la imposibilidad para otros de producir el producto, sería una barrera absoluta; la exclusividad, como condición límite de la diferenciación entre los sustitutivos, la que daría lugar a la creación de segmentos estancos, sería una barrera sólo relativa, disuasoria, si se combina con economías de escala en el segmento porque finalmente permitiría a otro u otros entrar aunque finalmente alguno deba, razonablemente irse.

\section{La interpretación de los sectores con diferenciación de productos con modelos espaciales}

Muchos mercados de productos de consumo -casi todos-, aunque no tengan una restricción logística derivada de la ubicación, son interpretables en clave espacial, con modelos similares al desarrollado en este artículo. Pero es necesario profundizar en todos los aspectos. ¿Qué clase de modelo espacial es aplicable, en cada caso, para interpretar la realidad?

Los profesionales de marketing de bienes de consumo emplean frecuentemente la palabra mapa -de innegable referencia espacial- para exponer la relación entre los atributos de sus productos y facilitar el posicionamiento de éstos - de nuevo la referencia espacial- frente a los diferentes segmentos - una vez más el símil espacial- de consumidores. Así, cada mercado en el que la diferenciación jugase un papel fundamental podría ser interpretado en clave geométrica, utilizando los atributos significativos de cada producto como variables. Pero se pueden distribuir en un plano; en una línea recta, abierta; a lo largo de una circunferencia, como en el caso que hemos visto, en el interior de un círculo, en la superficie de una esfera, en su interior, o en cualquier tipo de espacio de tres dimensiones o más.

Pero en estos modelos, con sus matices, las condiciones de equilibrio se establecerían de la misma forma que venimos comentando. Los mercados así estarán segmentados y los segmentos, que podrían o no, total o parcialmente estancos, serían susceptibles de ser atendidos con propuestas específicas diferenciadas en mayor o menor medida. El mercado sería interpretable separando a los diferentes grupos de productores que se dirigirán a segmentos diferenciados, poco o nada permeables entre sí, dependiendo de la elasticidad de sus rectas dd' respectivas. A su vez, dentro de cada segmento habría competidores también algo diferenciados o no, en competencia directa, en principio, con los más próximos. Dependiendo de la permeabilidad entre los grupos, es decir de la estanqueidad relativa de los mismos, habrá una mayor o menor influencia entre ellos, es decir, una mayor o menor influencia de los productos sustitutivos, y dependiendo de la diferenciación entre los que forman parte del grupo más cercano, más o menos poder de monopolio para cada uno dentro del grupo.Y de forma complementa-

${ }^{5}$ Harold Hotelling, Stability in Competition, Economic Journal, vol. 39, no. I 53 (March 1929), 4 I-57 
ria operaría la dificultad del acceso al segmento porque éste requiriera una condición de exclusividad, de singularidad alcanzable por uno o unos pocos.

Y, por ejemplo, una curiosidad que desarrollaremos en otro artículo: en el caso de la distribución a lo largo de una recta de los posibles clientes, se plantea una opción muy interesante que Hotelling ${ }^{6}$ analizó en su, también célebre, dilema. Si dos vendedores de perritos calientes se situaran en un playa de, pongamos por ejemplo, un kilómetro de longitud, lo más eficiente sería que cada uno atendiera a una mitad de la playa, situándose, cada uno de ellos, en el medio de su mitad. De esta manera se minimizarían los desplazamientos de los clientes: cada uno atendería a media playa. El desplazamiento máximo sería de un cuarto de kilómetro. Pero puesto que cada uno de ellos sabe que si se desplazara hacia el centro de la playa conservaría los clientes de su lado, seguros, y resultaría más atractivo para algunos de los otros, atendidos en principio de forma preferente por su competidor, se iría hacia el centro. Como el otro sabe lo mismo, al final ambos vendedores se situarían en el medio de la playa, juntos, lo que, pese a su lógica competitiva, no es eficiente desde el punto de vista económico. Este hecho está también, para nosotros, en la base de la teoría de los «clusters» que Porter ${ }^{7}$ analiza en su célebre diamante en el que las condiciones de la demanda, las condiciones de los factores, la presencia de industrias complementarias o relacionadas y la propia competencia entre las instaladas en la ubicación preferente provocan la aglomeración de las industrias.

\section{La publicidad como elemento diferenciador y su papel como modificador de la demanda}

A veces tratamos a los costes de publicidad como si se tratase de un coste más ligado a la producción, cuando tienen naturaleza muy diferente. La publicidad, en sentido estricto, en muchos casos, no va orientada a producir el producto sino a modificar las características de la demanda; la mayor parte de la inversión publicitaria no va orientada sólo a informar de que el producto existe, en este sentido sí sería un coste de producción sino a fidelizar a la clientela respecto a su consumo modificando la demanda.

Hay muchas variables que influyen en la mayor o menor presión publicitaria en cada sector. Es evidente que no es lo mismo un mercado de bienes de consumo que un mercado de bienes intermedios o bienes de producción; no es lo mismo un mercado de bienes de consumo de búsqueda previa que un mercado de bienes de consumo de experiencia, no es lo mismo un mercado con un número reducido de compradores que un mercado con un gran número de ellos.

La existencia de costes de ventas - publicidad y otrosque afectan a las curvas de demanda, invalidan los modelos micro-económicos clásicos, o, al menos relativizan su aplicabilidad práctica. Como señalábamos más arriba, la diferenciación entre los productos puede ser real -cuando los productos en el sector sean realmente diferentes para cada segmento y, por tanto, percibidos así- o ser sólo una diferenciación formal - cuando la marca consiga identificar a cada producto: diferenciarlo formalmente-. Con la identificación, cada segmento de consumidores se siente más atraído por los atributos formales de cada marca y se crea una relación de sustitución no perfecta entre las diferentes marcas en cada segmento.

En un sector de actividad de productos de consumo, que, en el fondo, sean poco diferenciables -esto es, que tengan elasticidades cruzadas muy significativas entre los competidores del grupo estratégico- se puede establecer una carrera de inversiones publicitarias por parte de los competidores principales -de hecho es muy común que esta carrera se establezca-, que, al cabo de poco tiempo, representaría la creación de enormes barreras por costes hundidos, necesarios para que los productos que compitan sean identificados, que impediría la entrada de competidores potenciales, puesto que cualquier nuevo entrante debería abordar fortísimas inversiones para lograr una imagen reconocible que le permitiera captar una parte suficiente del mercado. Muchas veces en los sectores de productos de consumo -en los que nos parecería por la estructura oligopolística de la oferta, que pudieran darse economías de escala, aunque en realidad no es así y éstas no serían tan relevantes- es ésta, y no otra, la razón de la constitución de un pequeño grupo de empresas competidoras que actúan, a la larga, como un cártel, aunque de forma transitoria se enreden en guerras de precios que pronto terminan en acuerdos colusorios tácitos, en cuanto reconocen su mutua dependencia.

La publicidad masiva dirigida a la creación de marcas, a la identificación formal de productos realmente in-

\footnotetext{
6 Michael E. Porter. "The Competitive Advantage of Nations", Free Press(1990, 1998).

7 Michael E. Porter. "The Competitive Advantage of Nations", Free Press (1990, 1998)
} 
diferenciados a través de la asignación de atributos más o menos artificiales, tiene tres efectos distintos sobre las curvas de demanda: de una parte modificar la demanda global del mercado de la categoría ${ }^{8}$, aumentándola; de otra parte aumentar la demanda particular de la marca que se anunciase a través del aumento del tamaño del segmento de mercado en el que la marca sería preferida a las otras -lo que sumado al efecto anterior podría provocar bien el aumento o bien la disminución de la demanda en los segmentos de mercado al alcance de cada uno de los demás concurrentes, esto es, de las demás marcas-; y, en tercer lugar, también, producir la disminución de la elasticidad de la demanda particular de la marca anunciada, es decir, incrementar su poder relativo de monopolio.

A cada precio, para un producto determinado en un mercado determinado, un coste de venta adicional produciría un incremento de la demanda y un incremento de los costes totales. La curva de costes totales se habría desplazado hacia la derecha y hacia arriba.

\section{I. La proliferación de marcas como barrera}

En algunos sectores de actividad se producen barreras derivadas de la publicidad de los instalados. Los productos de consumo tienen una serie de atributos formales que no es ilimitada. Por ejemplo un cereal para el desayuno puede ser rico, crujiente, sano, energético, divertido y, tal vez, alguna cosa más, pero no muchas más. Pues bien, si los concurrentes crearan diferentes marcas que se apropiaran, en la mente de los consumidores, de cada uno de estos atributos también disuadirían la entrada de competidores potenciales que no dispondrían de atributos formales de los que apoderarse.

\section{Conclusiones}

La estrategia de diferenciación sólo tiene sentido si hay segmentos en los que diferenciarse. No se puede plantear, porque sería un imposible metafísico, ser diferente para todos porque eso sería tanto como no ser diferente para nadie; incluso aunque se fuera único no se sería diferente.
Si hay segmentos en un mercado puede que estos sean estancos, o parcialmente estancos, pero en ningún caso esa estanqueidad mayor o menor garantiza que la estrategia de diferenciación que ese hecho impone sea generadora de ventajas; no hay otra posible, pero no es necesariamente generadora de beneficio porque restrinja la competencia. $\bigcirc$ en los segmentos estancos hay, por efecto de las economías de escala, un grupo pequeño que consciente de su mutua dependencia actúa como un cártel con un acuerdo colusorio sobre los precios, tácito o explícito, que hace que actúen como si fueran uno sólo en el segmento -y piénsese que siempre serán más eficaces las economías de escala como barrera en una parte que un todo- o el acceso a cada segmento está disuadido eficazmente porque la condición de exclusividad sea muy difícil o imposible de lograr, o no habrá ventaja para los que estén en el segmento aunque sean diferenciados.

\section{Bibliografía}

BAIN, J. (1956). Barriers to New Competition. Harvard University Press, Boston, MA, USA

BOWMAN, C., y FAULKNER, D. (1994).Measuring product advantages using competitive benchmarking and customer perceptions. Long Range Planning, Nº 27

CHAMBERLIN, EH. (1946). The Theory of Monopolistic Competition. Harvard University Press, Cambridge, MA, USA

LERNER, AP. (1934). The Concept of Monopoly and the Measurement of Monopoly Power.Review of Economic Studies

MATA, G. (2008).Las barreras para la entrada de competidores potenciales a los sectores de actividad y su influencia en la posibilidad de obtener beneficios en los mismos. Revista de Dirección, Organización y Administración de Empresas de La Fundación General de la Universidad Politécnica de Madrid, N 36, Octubre

MATA, G. (2008). Un nuevo modelo de clasificación de los entornos genéricos de las actividades empresariales. Revista de Economía y Derecho de la Sociedad de Economía y Derecho UPC, Universidad Peruana de Ciencias Aplicadas, $N^{\circ}$ 20. Primavera

MATA, G., y RODRÍGUEZ, C. (20 I0). El análisis estructural de los sectores de actividad: Análisis externo genérico, crítica del modelo de las cinco fuerzas de M.E. Por-

\footnotetext{
${ }^{8}$ Categoría: conjunto de bienes o servicios que se dirigen a satisfacer la misma necesidad.
} 
ter. Revista de Dirección, Organización y Administración de Empresas de La Fundación General de la Universidad Politécnica de Madrid, N. ${ }^{\circ}$ 40, Abril

PORTER, ME. (1980). Competitive Strategy.Free Press, New York, USA

PORTER, ME. ( 1 985). Competitive Advantage.Free Press, New York, USA
PORTER, ME. (1990). The Competitive Advantage of Nations. Free Press, New York, USA

SAFÓN,V., y ESCRIVÁ,A. (2002).Estrategias competitivas: implicaciones teóricas, prácticas y docentes. Revista Europea de Dirección y Economía de la Empresa, vol. I I, N. ${ }^{\circ} 2$. 